\title{
Unplanned Second-Stage Decompression for Neurological Deterioration Caused by Central Canal Stenosis after Indirect Lumbar Decompression Surgery
}

\author{
Hiroaki Nakashima ${ }^{1}$, Tokumi Kanemura ${ }^{1}$, Kotaro Satake ${ }^{1}$, Yoshimoto Ishikawa ${ }^{1}$, \\ Jun Ouchida ${ }^{1}$, Naoki Segi ${ }^{2}$, Hidetoshi Yamaguchi ${ }^{2}$, Shiro Imagama ${ }^{2}$ \\ ${ }^{1}$ Department of Orthopedic Surgery, Konan Kosei Hospital, Konan, Japan \\ ${ }^{2}$ Department of Orthopedic Surgery, Nagoya University Graduate School of Medicine, Nagoya, Japan
}

\begin{abstract}
Study Design: Prospective cohort study.
Purpose: This study aimed to identify risk factors for unplanned second-stage decompression for postoperative neurological deficit after indirect decompression using lateral lumbar interbody fusion (LLIF) with posterior fixation.

Overview of Literature: Indirect lumbar decompression with LLIF has been used as a minimally invasive alternative to direct decompression to treat degenerative lumbar diseases requiring neural decompression. However, evidence on the prevalence of neurological deficits caused by spinal canal stenosis after indirect decompression is limited.

Methods: This study included 158 patients (mean age, 71.13 \pm 7.98 years; male/female ratio, 67/91) who underwent indirect decompression with LLIF and posterior fixation. Indirect decompression was performed at 271 levels (mean level, 1.71 \pm 0.97 ). Logistic regression analysis was used to identify the risk factors for postoperative neurological deficits. The variables included were age, sex, body mass index, presence of primary diseases, diabetes mellitus, preoperative motor deficit, levels operated on, preoperative severity of lumbar stenosis, and preoperative Japanese Orthopedic Association (JOA) score.

Results: Postoperative neurological deficit due to spinal canal stenosis occurred in three patients (1.9\%). Spinal stenosis due to hemodialysis $(p<0.001)$, ligament ossification $(p<0.001)$, presence of preoperative motor paralysis $(p<0.001)$, low J0A score $(p=0.004)$, and severe canal stenosis $(p=0.02)$ were significantly more frequent in the paralysis group.

Conclusions: Severe preoperative canal stenosis and neurological deficit were identified as risk factors for postoperative neurological deterioration caused by spinal canal stenosis. Additionally, uncommon diseases, such as spinal stenosis due to hemodialysis and ligament ossification, increased the risk of postoperative neurological deficit; therefore, in such cases, indirect decompression is contraindicated.
\end{abstract}

Keywords: Lateral interbody fusion; Indirect decompression; Neurological deterioration; Complication; Lumbar stenosis 


\section{Introduction}

Lateral lumbar interbody fusion (LLIF) is a minimally invasive surgical technique used commonly as an alternative to conventional anterior lumbar interbody fusion (ALIF) or posterior lumbar interbody fusion (PLIF) [1]. LLIF permits maximal disc excision and endplate preparation for lumbar interbody fusion. Moreover, this procedure provides an effective increase in canal diameter and disc and neuroforaminal heights, thus resulting in indirect neural decompression [2-4]. This type of indirect decompression has several advantages over PLIF, including lower risk of direct neural injury, incidental durotomy, and postoperative perineural fibrosis; decreased blood loss; and minimal surgical invasiveness $[4,5]$. Furthermore, LLIF also reduces the risk of major visceral/vessel injuries typically observed with ALIF [5]. Therefore, indirect decompression with LLIF is commonly used to treat patients with axial lower back pain or degenerative lumbar diseases requiring neural decompression [4].

However, surgical indications for indirect decompression with LLIF remain fairly debatable. Although indirect decompression with LLIF has been recommended for treatment of mild canal stenosis [1], its efficacy and safety in case of severe canal stenosis remain undetermined. Fujibayashi et al. [4] examined 28 patients who underwent indirect decompression using LLIF and reported that the procedure resulted in sufficient neural decompression and clinical improvement in all patients, including those exhibiting severe stenosis. Furthermore, greater improvements in neural decompression were observed in patients with more severe preoperative stenosis than in those with milder stenosis, suggesting that indirect decompression was more effective in the former group of patients. However, indirect decompression may also increase the risk of complications, such as the intraoperative neurological deficit caused by spinal canal stenosis, particularly in cases in which correction of spondylolisthesis or spinal alignment was performed without direct neural decompression in the presence of a severely narrow canal.

Although several studies have reported the occurrence of motor paralysis caused by deficit to the iliopsoas muscle or lumbar plexus nerve injury in patients undergoing LLIF [6], the prevalence of neurological deficits caused by spinal_canal stenosis among these patients remains unclear. LLIF has been used commonly for more challenging cases, including deformities or severe stenosis, and this complication could have occurred during the course of indirect decompression. However, a limited number of studies focus on the occurrence of this complication, and its risk factors are largely unknown.

Therefore, the key objectives of this study included (1) assessment of the prevalence of perioperative neurological deterioration caused by spinal canal stenosis during indirect decompression with LLIF, (2) analysis of the risk factors associated with this neurological deficit, and (3) identification of surgical contraindications for use of indirect decompression for treatment of degenerative lumbar diseases.

\section{Materials and Methods}

We prospectively reviewed surgical and radiographic data of individuals diagnosed with degenerative lumbar diseases requiring neural decompression and surgical intervertebral fusion at one institution. Ethical approval was obtained from the Konan Kosei Hospital institutional ethics comittes (IRB approved no., 25-022[0174]). Patients underwent indirect neural decompression with LLIF and surgical posterior pedicle screw fixation between October 2013 and January 2017. Patients with a primary surgical indication of spinal correction instead of indirect neural decompression were excluded from this study. The final sample comprised 158 consecutive patients (mean age, $71.13 \pm 7.98$ years; male/female ratio, 67/91), and indirect decompression was performed at 271 levels (mean level, $1.71 \pm 0.97)$. Among the procedures, 20 were performed at L1-L2, 44 at L2-L3, 83 at L3-L4, and 111 at L4-L5. Furthermore, 122 patients underwent extremely lateral lumbar fusion using Coroent cages (NuVasive Inc., San Diego, CA, USA), and 36 underwent oblique lumbar interbody fusion using Clydesdale cages (Medtronic Inc., Minneapolis, MN, USA) for LLIF. Percutaneous or conventional open posterior pedicle screw instrumentation was performed without direct posterior neural decompression in all patients.

To distinguish from proximal muscle weakness caused by injury to the lumbosacral plexus or iliopsoas muscle, neurological deficit secondary to spinal_canal stenosis following indirect decompression was defined as the occurrence of bilateral motor paralysis and/or urinary/bowel dysfunction that could not be explained by a single nerve root injury within 1 week postoperatively.

Demographic data collected included age, sex, pres- 
ence of diabetes mellitus (DM), presence of other primary diseases, number of operated levels, spinal levels of indirect decompression, preoperative Japanese Orthopedic Association (JOA) score, presence of preoperative motor paralysis (manual muscle test $[\mathrm{MMT}] \leq 3$ ), and severity of preoperative lumbar spinal stenosis, which was qualitatively scored using T2-weighted axial magnetic resonance imaging and the modified Schizas' classification [7] (Fig. 1). The three grades of this classification were as follows: grade A stenosis: visible, nonhomogeneously distributed cerebrospinal fluid inside the dural sac; grade B stenosis: no detectable rootlets, no cerebrospinal fluid signal visible in the dural gray signal, and no epidural fat present posteriorly; grade C stenosis: no identifiable rootlets and no evidence of epidural fat posteriorly.

Data are presented as mean \pm standard deviation, and chi-square and Student $t$-tests were used to compare the two groups. Risk factors associated with postoperative neurological deficit were identified using logistic regression analysis. Factors with $p<0.10$ on univariate analysis were included in the multivariate analysis. Statistical significance was set at $p<0.05$, and all statistical analyses were performed using IBM SPSS ver. 21.0 (IBM Corp., Armonk, NY, USA).
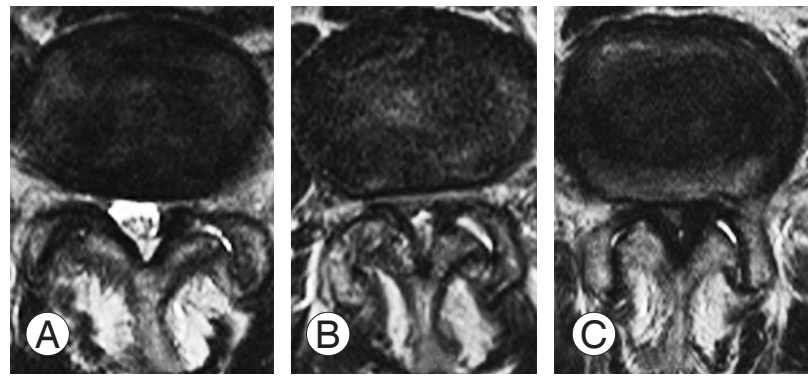

Fig. 1. Representative images of thecal sac stenosis, graded (A-C) using the modified Schizas' classification.

\section{Results}

The primary diseases exhibited by the patients included degenerative spondylolisthesis $(\mathrm{n}=105)$, degenerative kyphosis/scoliosis $(\mathrm{n}=27)$, adjacent segment disease $(\mathrm{n}=11)$, recurrent stenosis after direct decompression $(n=10)$, compression fracture with canal stenosis $(\mathrm{n}=3)$, ligament ossification around the spinal canal $(\mathrm{n}=1)$, and spinal stenosis due to hemodialysis and spondylolytic spondylolisthesis $(\mathrm{n}=1)$. The mean preoperative JOA score was $13.6 \pm 4.5$ points, and preoperative motor paralysis (MMT $\leq 3$ ) was observed in nine patients (5.7\%). DM and compression fractures at the surgical site were noted in 31 and 12 patients, respectively. Preoperative modified Schiza grades A, B, and C were noted at 32, 45, and 16 levels, respectively.

Three patients with primary diagnoses of ossification of the posterior longitudinal ligament (OPLL), spinal stenosis due to hemodialysis, and spinal stenosis with compression fracture exhibited postoperative neurological deficit caused by suspected spinal canal stenosis and underwent direct decompression. Two of these patients underwent a two-staged procedure (LLIF followed by posterior fixation 1 week later) and exhibited bilateral motor paralysis immediately following the posterior surgery. The third patient underwent surgery in a single stage and presented with an inability to urinate postoperatively (Table 1). All three patients exhibited preoperative motor paralysis $(\mathrm{MMT} \leq 3)$ in the lower extremities. Direct decompression was performed soon after neurological deterioration, and the patients exhibited gradual improvement thereafter.

Upon comparing patients with and without neurological deterioration, no significant differences in age $(p=0.59)$, sex ( $\mathrm{p}=0.39)$, body mass index (BMI, $p=0.11)$, number of operated levels ( $p=0.61$ ), and levels of indirect decompression ( $p=0.10-0.89)$ were observed (Table 2$)$. Spinal steno-

Table 1. Summary of patients with postoperative neurological deterioration

\begin{tabular}{|c|c|c|c|c|c|c|c|}
\hline Case & $\begin{array}{l}\text { Age } \\
\text { (yr) }\end{array}$ & Primary disease & $\begin{array}{c}\text { Preoperative } \\
\text { Japanese } \\
\text { Orthopedic } \\
\text { Association score }\end{array}$ & $\begin{array}{c}\text { Preoperative } \\
\text { manual } \\
\text { muscle test }\end{array}$ & $\begin{array}{c}\text { Schizas' } \\
\text { classification }\end{array}$ & $\begin{array}{l}\text { No. of } \\
\text { levels } \\
\text { operated } \\
\text { on }\end{array}$ & $\begin{array}{l}\text { Neurological } \\
\text { deterioration }\end{array}$ \\
\hline 1 & 65 & $\begin{array}{l}\text { Ossification of ligament around } \\
\text { spinal canal }\end{array}$ & 7 & 3 & C & 2 & Bilateral motor \\
\hline 2 & 57 & Spinal stenosis due to hemodialysis & 5 & 3 & B & 2 & Bilateral motor \\
\hline 3 & 84 & $\begin{array}{l}\text { Degenerative spondylolisthesis } \\
\text { with compression fracture }\end{array}$ & 7 & 3 & C & 2 & Urinary incontinence \\
\hline
\end{tabular}


Table 2. Comparison of factors in patients with and without postoperative neurological deficit

\begin{tabular}{|c|c|c|c|}
\hline Variable & Postoperative paralysis (-) & Postoperative paralysis (+) & $p$-value \\
\hline No. of patients & 155 & 3 & \\
\hline \multicolumn{4}{|l|}{ Background } \\
\hline Age (yr) & $71.2 \pm 8.0$ & $68.7 \pm 13.9$ & 0.59 \\
\hline Sex (male/female) & $65 / 90$ & $2 / 1$ & 0.39 \\
\hline Body mass index $\left(\mathrm{kg} / \mathrm{m}^{2}\right)$ & $24.5 \pm 3.6$ & $28.1 \pm 11.4$ & 0.11 \\
\hline No. of levels operated on & $1.7 \pm 1.0$ & $2.0 \pm 0.0$ & 0.61 \\
\hline \multicolumn{4}{|l|}{ Primary diseases } \\
\hline Degenerative spondylolisthesis & 104 & 1 & 0.22 \\
\hline Degenerative kyphosis/scoliosis & 27 & 0 & 0.43 \\
\hline Adjacent segment disease & 11 & 0 & 0.63 \\
\hline Recurrent stenosis after direct decompression & 10 & 0 & 0.65 \\
\hline Spinal stenosis due to hemodialysis & 2 & 1 & $<0.001^{*}$ \\
\hline Ossification of ligament around spinal canal & 1 & 1 & $<0.001^{*}$ \\
\hline Spondylolytic spondylolisthesis & 1 & 0 & 0.89 \\
\hline \multicolumn{4}{|l|}{ Other diseases } \\
\hline Diabetes mellitus & 30 & 1 & 0.55 \\
\hline Compression fractures at the levels operated on & 11 & 1 & 0.09 \\
\hline \multicolumn{4}{|l|}{ Levels of indirect decompression } \\
\hline $\mathrm{L} 1 / 2$ & 20 & 0 & 0.51 \\
\hline $\mathrm{L} 2 / 3$ & 43 & 1 & 0.83 \\
\hline $\mathrm{L} 3 / 4$ & 80 & 3 & 0.10 \\
\hline$\llcorner 4 / 5$ & 109 & 2 & 0.89 \\
\hline \multicolumn{4}{|l|}{ Severity of preoperative neurological disorder } \\
\hline Preoperative paralysis of manual muscle test $\leq 3$ & 6 & 3 & $<0.001^{*}$ \\
\hline Preoperative Japanese Orthopedic Association score & $13.8 \pm 4.4$ & $6.3 \pm 1.2$ & $0.004^{*}$ \\
\hline \multicolumn{4}{|l|}{ Severity of stenosis } \\
\hline Schizas' classification A & 57 & 0 & 0.19 \\
\hline Schizas' classification B & 75 & 1 & 0.61 \\
\hline Schizas' classification C & 23 & 2 & $0.02^{*}$ \\
\hline
\end{tabular}

Values are presented as number or mean \pm standard deviation, unless otherwise stated. * $p<0.05$ (statistically significant differences).

sis due to hemodialysis $(p<0.001)$, OPLL $(p<0.001)$, and preoperative motor paralysis (MMT $\leq 3, p<0.001)$ were significantly more frequent among patients with postoperative neurological deterioration. Preoperative JOA score was significantly lower $(6.3 \pm 1.2)$ among patients with postoperative neurological deficits than those without deficits (13.8 $\pm 4.4, p=0.004)$. With regard to preoperative canal stenosis severity, the modified Schizas' classification grade $\mathrm{C}$ occurred significantly more frequently $(p=0.02)$ among patients with neurological deterioration than the other grades (A and B).
Spinal stenosis due to hemodialysis (odds ratio [OR],

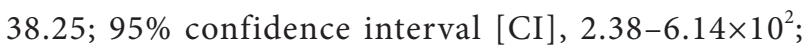
$p=0.01$ ), preoperative JOA score (OR, 0.54; $95 \% \mathrm{CI}$, $0.32-0.94 ; p=0.03$ ), and preoperative modified Schizas' classification grade C (OR, 11.48; 95\% CI, $1.00-1.31 \times 10^{2}$; $p=0.05)$ exhibited $p$-values $<0.10$ on univariate analysis (Table 3). However, upon inclusion of these variables in the multivariate analysis, no statistically significant differences were observed (Table 3 ).

A 65 -year-old, obese $\left(B M I=40.9 \mathrm{~kg} / \mathrm{m}^{2}\right)$ woman presented with pain in the right buttock and lower limb (case 
Table 3. Multivariate logistic regression analysis of risk factors associated with postoperative neurological deficit

\begin{tabular}{|c|c|c|c|c|}
\hline \multirow{2}{*}{ Variable } & \multicolumn{2}{|c|}{ Univariate analysis } & \multicolumn{2}{|c|}{ Multivariate analysis } \\
\hline & $\mathrm{OR}(95 \% \mathrm{CI})$ & $p$-value & OR $(95 \% \mathrm{CI})$ & $p$-value \\
\hline \multicolumn{5}{|l|}{ Background } \\
\hline Age (yr) & $0.96(0.85-1.10)$ & 0.59 & & \\
\hline Sex (male/female) & $2.77(0.25-31.19)$ & 0.41 & & \\
\hline Body mass index $\left(\mathrm{kg} / \mathrm{m}^{2}\right)$ & $1.19(0.96-1.47)$ & 0.12 & & \\
\hline No. of operated levels & $1.31(0.46-3.74)$ & 0.61 & & \\
\hline \multicolumn{5}{|l|}{ Primary diseases } \\
\hline Degenerative spondylolisthesis & $0.25(0.02-2.77)$ & 0.26 & & \\
\hline Degenerative kyphosis/scoliosis & $0.00(0.00)$ & 1.00 & & \\
\hline Adjacent segment disease & $0.00(0.00)$ & 1.00 & & \\
\hline Recurrent stenosis after direct decompression & $0.00(0.00)$ & 1.00 & & \\
\hline Spinal stenosis due to hemodialysis & $38.25\left(2.38-6.14 \times 10^{2}\right)$ & $0.01^{*}$ & $3.01 \times 10^{11}(0.00)$ & 1.00 \\
\hline Ossification of ligament around spinal canal & $77.00(3.46-1,714.37)$ & $0.006^{*}$ & $2.20 \times 10^{7}(0.00)$ & 1.00 \\
\hline Spondylolytic spondylolisthesis & $0.00(0.00)$ & 1.00 & & \\
\hline \multicolumn{5}{|l|}{ Other diseases } \\
\hline Diabetes mellitus & $0.48(0.04-5.47)$ & 0.55 & & \\
\hline Compression fracture at the levels operated on & $6.55(0.55-77.97)$ & 0.14 & & \\
\hline \multicolumn{5}{|l|}{ Levels of indirect decompression } \\
\hline $\mathrm{L} 1 / 2$ & $0.00(0.00)$ & 1.00 & & \\
\hline $\mathrm{L} 2 / 3$ & $1.30(0.12-14.74)$ & 0.83 & & \\
\hline $\mathrm{L} 3 / 4$ & $6.06 \times 10^{7}(0.00)$ & 1.00 & & \\
\hline$\llcorner 4 / 5$ & $0.84(0.08-9.54)$ & 0.89 & & \\
\hline \multicolumn{5}{|l|}{ Severity of preoperative neurological disorder } \\
\hline Preoperative paralysis of manual muscle test $\leq 3$ & $6(0.00)$ & 1.00 & & \\
\hline Preoperative Japanese Orthopedic Association score & $0.54(0.32-0.94)$ & $0.03^{*}$ & $0.46(0.11-1.93)$ & 0.29 \\
\hline \multicolumn{5}{|l|}{ Severity of stenosis } \\
\hline Schizas' classification A & $0.00(0.00)$ & 1.00 & & \\
\hline Schizas' classification B & $0.53(0.05-6.00)$ & 0.61 & & \\
\hline Schizas' classification C & $11.48\left(1.00-1.31 \times 10^{2}\right)$ & 0.05 & $4.45 \times 10^{11}(0.00)$ & 1.00 \\
\hline
\end{tabular}

$\mathrm{OR}$, odds ratio; $\mathrm{Cl}$, confidence interval.

${ }^{*} p<0.05$ (statistically significant differences).

1 in Table 1) with several comorbidities, including hypertension and severe asthma (forced expiratory volume percentage per one second, $66.3 \%$ ). She also exhibited frequent urination and partial lower limb paralysis preoperatively (MMT value of tibialis anterior [TA], 3/5; gastrocutaneous $[\mathrm{GC}], 3 / 5$; extensor halluces longus [EHL], 3/5; and flexor halluces longus [FHL], 3/5). Computed tomography revealed ossification of the yellow ligament and modified Schizas' classification grade C lumbar spinal stenosis at L2-L3 and L3-L4. Indirect decompression was selected as the treatment of choice taking her obesity and comorbidities into consideration (Fig. 2A-C). She underwent LLIF at L2-L3 and L3-L4, and her pain and paralysis decreased (TA, 3 to 4; EHL, 2 to 3) post-LLIF (Fig. 2D, E). Despite undergoing percutaneous pedicle screw instrumentation 6 days after LLIF, her postoperative MMT values for TA, GC, EHL, and FHL were zero (Fig. $2 \mathrm{~F})$. Therefore, posterior direct decompression surgery was performed as soon as possible after posterior instrumentation, and her paralysis partially improved thereafter. 


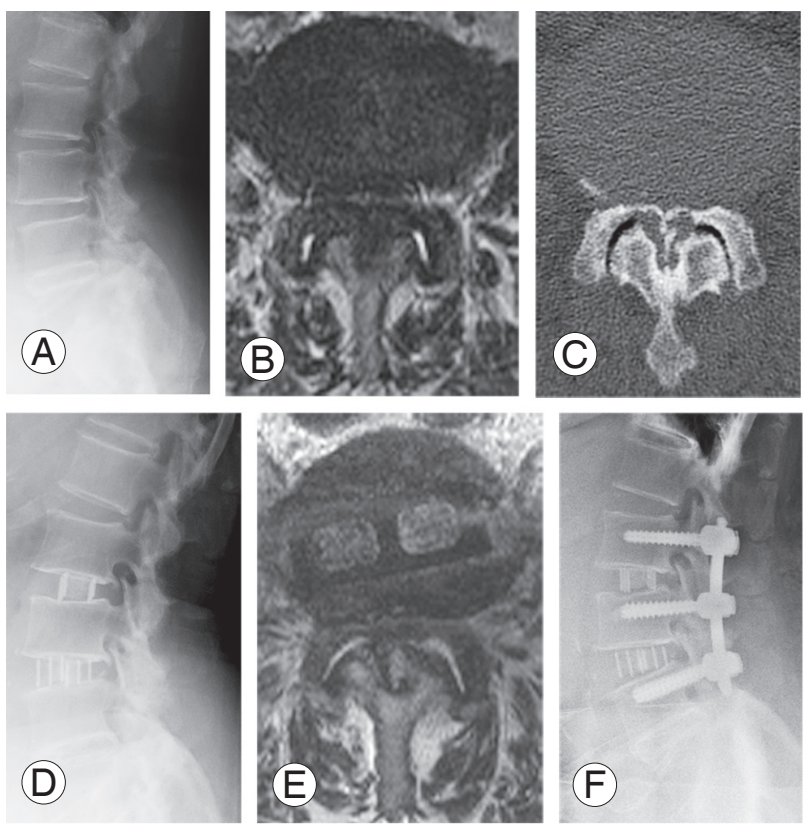

Fig. 2. Representative images of case 1 (with postoperative neurological deficit). (A) Preoperative lateral X-ray; (B) preoperative T2-weighted axial MRI; (C) preoperative axial computed tomography image at L4-5; (D) lateral X-ray after LLIF; (E) T2-weighted axial MRI after LLIF; (F) postoperative $X$-ray after percutaneous pedicle screw fixation. MRI, magnetic resonance image; LLIF, lateral lumbar interbody fusion.

\section{Discussion}

To our knowledge, this is the first study to assess the risk factors and degree of postoperative neurological deterioration caused by suspected spinal canal stenosis among patients undergoing indirect decompression using LLIF and posterior instrumentation. Our results demonstrated that patients with preoperative lower limb paralysis, low JOA scores, and severe stenosis were at a higher risk of perioperative neurological deterioration and that this was particularly true for patients exhibiting ligament ossification around the spinal canal or spinal stenosis due to hemodialysis.

Ozgur et al. [1] first introduced extreme LLIF for treatment of axial lower back pain, and Oliveira et al. [2] developed indirect decompression using LLIF for spinal canal or foraminal stenosis. Indirect lumbar decompression after LLIF can be achieved by reduction of disc bulging and elongation of the hypertrophied ligamentum flavum via restoration of disc height [4]. Radiographic studies have demonstrated significant increases in the foraminal area (26\%-35\%), dural sac area (up to $143 \%$ ), and posterior disc height $(13 \%-20 \%)$ in patients with mild-to-moderate stenosis $[2,8,9]$. However, the degree of indirect decompression in cases of severe spinal canal stenosis, significant rotatory scoliosis, and moderateto-severe spondylolisthesis was unclear; based on our results, it appears that this surgery was contraindicated in such cases [1]. More recently, LLIF has been used widely to treat moderate spondylolisthesis, severe stenosis, and adult degenerative lumbar scoliosis [4,10-13], although the indications for indirect decompression remain the subject of debate. The majority of studies report neurological improvement following indirect decompression with LLIF in selected patients. However, considering patients with severe spinal canal stenosis were excluded from these studies, the efficacy of this procedure in such cases is unclear. Fujibayashi et al. [4] reported satisfactory clinical results, including greater neural decompression, following surgery in patients with severe stenosis compared with those with mild-to-moderate stenosis. Sembrano et al. [14] conducted a prospective study that compared minimally invasive transforaminal and lateral interbody fusion for treatment of low-grade degenerative spondylolisthesis; they reported that similar clinical recovery was achieved with both surgical techniques irrespective of preoperative neurological symptom severity.

However, unplanned second-stage decompression was required in some patients after indirect decompression. Oliveira et al. [2] reported that two (9.5\%) of 21 patients studied required unplanned direct decompression, and they concluded that indirect decompression with LLIF may not adequately decompress the spinal canal in patients exhibiting osteophytes in the lateral recesses or those with foraminal stenosis. Malham et al. [9] reported that unplanned second-stage decompression was required in $11(9.0 \%)$ of 122 patients who underwent indirect decompression because they exhibited persistent or newly developed radicular leg pain $<6$ months postoperatively. They performed additional surgeries because of insufficient clinical recovery or iatrogenic leg pain due to cage misplacement.

Although it has been reported that unplanned secondstage decompression was required in some patients after indirect decompression, to our knowledge, no study has reported unplanned second-stage decompression for neurological deterioration caused by suspected spinal canal stenosis. Although we initiated indirect decompression with LLIF from mild stenosis for treating lowgrade degenerative spondylolisthesis, we experienced 
this complication in the expansion of clinical indications. Even though indirect decompression could be performed without any complications in the majority of cases with severe stenosis, postoperative neurological deterioration occurred in those with severe preoperative neurological deficit, such as paralysis, rare diseases such as spinal stenosis due to hemodialysis, or ligament ossification around the spinal canal. Amyloid deposition [15] or ligament ossification occurred in patients with spinal stenosis due to hemodialysis or OPLL, and the pathology in the ligament differed considerably from that of hypertrophy caused by degeneration. Consequently, indirect decompression should not be indicated in such cases because preserving the spinal ligament is difficult while performing the procedure.

Additionally, postoperative neurological deficit occurred after posterior instrumentation instead of after LLIF in two of the cases (cases 1 and 2 in Table 1). Although the timing of paralysis was unclear in the other case (case 3 in Table 1), in which LLIF and posterior instrumentation were performed on the same day, it is conceivable that perioperative spinal alignment change due to posterior instrumentation may induce neurological deficit in cases with severe stenosis. Supplementation of LLIF with pedicle screw fixation has been reported to provide greater stabilization, reduce cage subsidence, and result in better bony fusion $[4,12]$. However, posterior instrumentation may also increase lumbar lordosis, resulting in paralysis. In the present study, severe preoperative stenosis, severe preoperative neurological deficit, low JOA score, and motor paralysis were significant risk factors for paralysis. We did not perform intraoperative spinal alignment correction; the spinal alignment resulted from intraoperative surgical position. It is extremely dangerous to perform posterior instrumentation without direct decompression in patients with damaged cauda equina and severe stenosis exhibiting preoperative paralysis. However, considering that indirect lumbar decompression can be achieved by re-stretching the yellow ligamentum, we should note that there could be a tradeoff between indirect lumbar decompression and adequate lumbar lordosis; thus, we should be particularly careful in cases of severe stenosis. Unfortunately, intraoperative neuromonitoring was not performed during posterior fixation in the three cases with perioperative neurological deficit; therefore, the time at which the deficit occurred during surgery was unclear. Further verification is necessary.
Following the observation of postoperative paralysis in three cases, indirect decompression only was adopted for patients without neurological symptoms, such as paralysis or severe leg pain at rest. No postoperative paralysis occurred thereafter in $>200$ cases. No evidence of postoperative neurological deterioration has been observed since, even in patients with severe stenosis (grade $\mathrm{C}$, modified Schizas' classification). Indirect decompression should be considered seriously as a treatment option in patients with severe stenosisThis study had certain limitations. First, it evaluated a relatively small number of cases with postoperative paralysis, thus resulting in relatively low statistical power in the multivariate analysis. Because of the small number (three patients), their diagnosis and comorbidities were identified as significant risk factors. Future large scale prospective studies are necessary to validate this result. Second, the postoperative neurological deterioration was considered to result from spinal canal stenosis as there was bilateral paralysis in multiple muscles or urinary/bowel dysfunction. However, a definitive diagnosis of this was difficult, and so other possible causes of the paralysis cannot be excluded. Nevertheless, the neurological deficit improved after direct decompression in all three cases, suggesting that spinal canal stenosis was the most likely cause.

\section{Conclusions}

An increased risk of perioperative neurological deficit was observed in cases of severe preoperative canal stenosis and neurological deficit, particularly in spinal stenosis due to hemodialysis or OPLL. Therefore, indirect lumbar decompression with LLIF and posterior instrumentation should not be considered in such high-risk patients.

\section{Conflict of Interest}

No potential conflict of interest relevant to this article was reported.

\section{References}

1. Ozgur BM, Aryan HE, Pimenta L, Taylor WR. Extreme lateral interbody fusion (XLIF): a novel surgical technique for anterior lumbar interbody fusion. Spine J 2006;6:435-43.

2. Oliveira L, Marchi L, Coutinho E, Pimenta L. A ra- 
diographic assessment of the ability of the extreme lateral interbody fusion procedure to indirectly decompress the neural elements. Spine (Phila Pa 1976) 2010;35:S331-7.

3. Pawar AY, Hughes AP, Sama AA, Girardi FP, Lebl DR, Cammisa FP. A comparative study of lateral lumbar interbody fusion and posterior lumbar interbody fusion in degenerative lumbar spondylolisthesis. Asian Spine J 2015;9:668-74.

4. Fujibayashi S, Hynes RA, Otsuki B, Kimura H, Takemoto M, Matsuda S. Effect of indirect neural decompression through oblique lateral interbody fusion for degenerative lumbar disease. Spine (Phila Pa 1976) 2015;40:E175-82.

5. Epstein NE. Extreme lateral lumbar interbody fusion: do the cons outweigh the pros? Surg Neurol Int 2016;7:S692-700.

6. Ahmadian A, Deukmedjian AR, Abel N, Dakwar E, Uribe JS. Analysis of lumbar plexopathies and nerve injury after lateral retroperitoneal transpsoas approach: diagnostic standardization. J Neurosurg Spine 2013;18:289-97.

7. Schizas C, Theumann N, Burn A, et al. Qualitative grading of severity of lumbar spinal stenosis based on the morphology of the dural sac on magnetic resonance images. Spine (Phila Pa 1976) 2010;35:191924.

8. Elowitz EH, Yanni DS, Chwajol M, Starke RM, Perin NI. Evaluation of indirect decompression of the lumbar spinal canal following minimally invasive lateral transpsoas interbody fusion: radiographic and outcome analysis. Minim Invasive Neurosurg 2011;54:201-6.

9. Malham GM, Parker RM, Goss B, Blecher CM. Clini- cal results and limitations of indirect decompression in spinal stenosis with laterally implanted interbody cages: results from a prospective cohort study. Eur Spine J 2015;24 Suppl 3:339-45.

10. Phillips FM, Isaacs RE, Rodgers WB, et al. Adult degenerative scoliosis treated with XLIF: clinical and radiographical results of a prospective multicenter study with 24-month follow-up. Spine (Phila Pa 1976) 2013;38:1853-61.

11. Isaacs RE, Hyde J, Goodrich JA, Rodgers WB, Phillips FM. A prospective, nonrandomized, multicenter evaluation of extreme lateral interbody fusion for the treatment of adult degenerative scoliosis: perioperative outcomes and complications. Spine (Phila Pa 1976) 2010;35:S322-30.

12. Castellvi AE, Nienke TW, Marulanda GA, Murtagh $\mathrm{RD}$, Santoni BG. Indirect decompression of lumbar stenosis with transpsoas interbody cages and percutaneous posterior instrumentation. Clin Orthop Relat Res 2014;472:1784-91.

13. Baghdadi YM, Larson AN, Dekutoski MB, et al. Sagittal balance and spinopelvic parameters after lateral lumbar interbody fusion for degenerative scoliosis: a case-control study. Spine (Phila Pa 1976) 2014;39:E166-73.

14. Sembrano JN, Tohmeh A, Isaacs R; SOLAS Degenerative Study Group. Two-year comparative outcomes of MIS lateral and MIS transforaminal interbody fusion in the treatment of degenerative spondylolisthesis: part I: clinical findings. Spine (Phila Pa 1976) 2016;41 Suppl 8:S123-32.

15. Fukutake T, Takagi K, Kuwabara S, et al. Destructive spondyloarthropathy of the cervical spine in hemodialyzed patients. No To Shinkei 1997;49:713-22. 